\title{
Advantages of Direct Detection and Electron Counting for High-Energy Resolution and Monochromated Electron Energy Loss Spectroscopy Data Acquisition
}

\author{
Paolo Longo $^{1}$, Ray D. Twesten ${ }^{1}$ and J. Alfredo Carranco Rodriguez ${ }^{2}$ and Gianluigi Botton ${ }^{2}$ \\ ${ }^{1 .}$ Gatan Inc., Pleasanton, CA. \\ 2. Department of Materials Science and Engineering, McMaster University, Hamilton, Canada.
}

Transmission electron microscopes primarily employ indirect cameras (IDC) for electron detection in imaging, diffraction and EELS modes. Such cameras convert incident electrons to photons which, through a fiber optic network or lens, are coupled to a light sensitive camera. This indirect detection method typically has a negative impact on the point spread function (PSF) and detective quantum efficiency (DQE) of the camera. Over the last decade, radiation tolerant CMOS active pixel sensors, which directly detect high-energy incident electrons and have the speed to count individual electrons events, have been developed. These detectors result in greatly improved PSF and DQE in comparison to conventional IDCs. Such direct detection cameras (DDCs) have revolutionized the cryo-TEM field as well as have strong advantages for in-situ TEM in both imaging and diffraction applications. It's already been shown that EELS applications can benefit from the improved PSF and the ability to count electrons. The improved PSF allows spectra to be acquired over larger energy ranges (low spectrometer dispersions) while maintaining sharp features and greatly reduced spectral tails. The ability to count electrons nearly eliminates the noise associated with detector readout and greatly reduces the proportional noise associated with detector gain variations. This effectively leaves the shot noise as the limiting noise source present. The implication for EELS acquisition is that fine structure analysis becomes more straightforward for typical conditions and even possible for the case of low signal levels. Here, we want to explore the effects of electron counting in the case of monochromated experiments where higher energy resolution is required.

As example of the advantages due to the reduced noise and PSF in the case of monochromated experiments, Figures 1c,d show the EELS spectra of Fe $\mathrm{L}_{2,3}$-edges at $708 \mathrm{eV}$ and $\mathrm{Ti} \mathrm{L}_{2,3}$-edges at $456 \mathrm{eV}$ extracted from the $\mathrm{Fe}_{2} \mathrm{O}_{3}$ and the $\mathrm{SrTiO}_{3}$ areas within the green box region in the ADF STEM survey image in Figure 1b. Spectra were extracted in STEM mode and the probe was rastered across the $\mathrm{SrTiO}_{3} / \mathrm{Fe}_{2} \mathrm{O}_{3}$ as shown in Figure 1b. The monochromator was excited to deliver an energy resolution of $0.1 \mathrm{eV}$ as shown in the low-loss spectrum in Figure 1a. The spectrum was acquired using the K2 (the DD detector for the experiment) in counting mode and the spectrometer setup with a dispersion of $0.1 \mathrm{eV} /$ channel that results in a spectrum with an energy range of $\sim 400 \mathrm{eV}$. In this way, both the Ti Ledges at 456 and the Fe L-edges at $708 \mathrm{eV}$ are present in the same spectrum. Every detail in both the Ti L and the Fe L near edge fine structure can be observed leading to a more accurate and precise chemical analysis and comparison with the results from simulations. Figures 2 show the comparison between spectra acquired using the $\mathrm{K} 2$ and $\mathrm{CCD}$ (the IDC detector used for the experiment) with the spectrometer setup for both experiments with a dispersion of $0.1 \mathrm{eV} /$ channel. The monochromator was excited exactly in the same way resulting in an energy resolution of $0.1 \mathrm{eV}$ measured using the $\mathrm{K} 2$ as shown in the low-loss spectrum in Figure 1a. In the case of the CCD, a dispersion of $0.1 \mathrm{eV} / \mathrm{channel}$ results in a spectrum with an energy range of $200 \mathrm{eV}$, thus the contribution from the Ti $\mathrm{L}_{2,3}$-edges at 456 is now missing. In the case of the $\mathrm{K} 2$, given the much reduced PSF, the energy resolution is such that the split of the $\mathrm{Fe}_{3}$ peak is very pronounced whereas in the case of the CCD the split is just a shoulder, higher energy resolution would be needed in order to cope with the high PSF and give enough energy 
resolution for chemical analysis. All these data was acquired using exactly the same experimental conditions across similar areas in the same specimen.

In this presentation, we will review the current state of electrons counting detectors for electron microscopy with an emphasis on system for high-energy resolution EELS measurements.
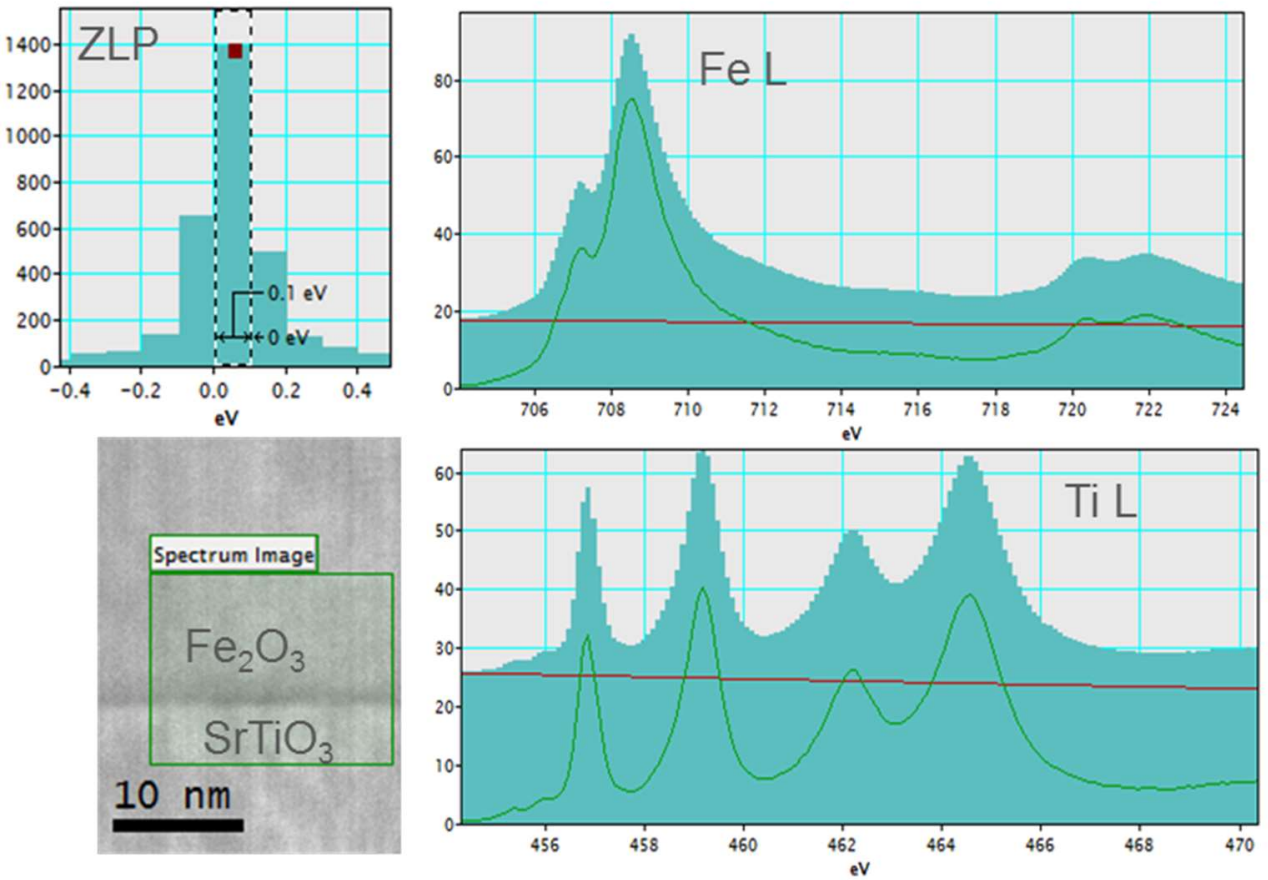

Figures 1. All data extracted using the $\mathrm{K} 2$ as detector. a) low-loss spectrum showing an energy resolution for the experiment of $0.1 \mathrm{eV}$ achieved with a dispersion of $0.1 \mathrm{eV} / \mathrm{channel}$; $\mathrm{b}$ ) ADF STEM survey image, the green region is where EELS spectra were collected point by point; c,d) EELS spectra of Fe L- and Ti L-edges at $708 \mathrm{eV}$ and $456 \mathrm{eV}$ extracted from the $\mathrm{Fe}_{2} \mathrm{O}_{3}$ and the $\mathrm{SrTiO}_{3}$ regions inside the green box in Figure 1b. Both the Fe and Ti L signals are extracted from the same spectrum.
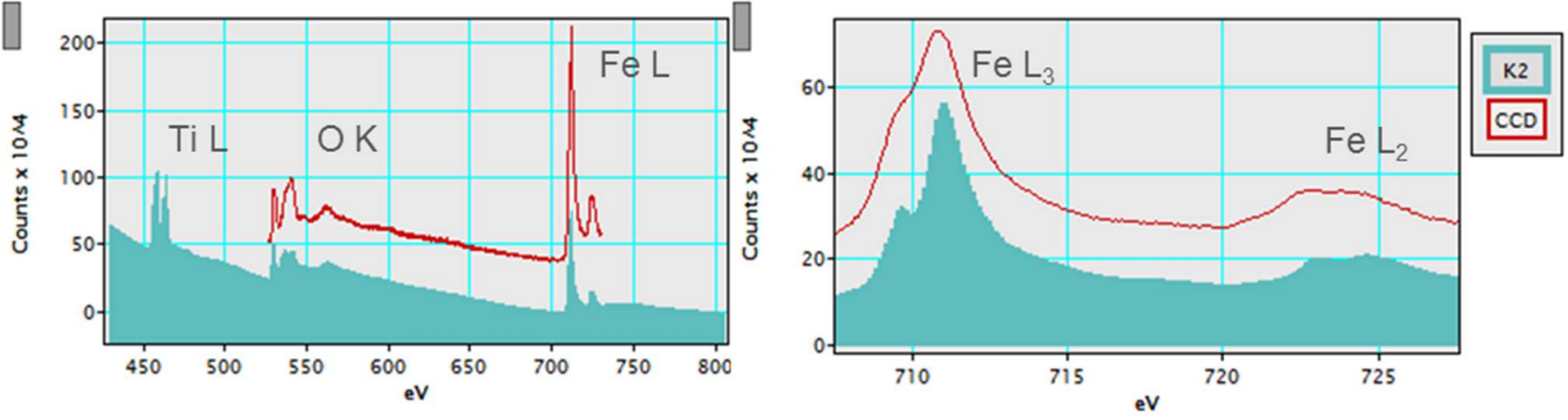

Figures 2. a) EELS spectra extracted using the $\mathrm{K} 2$ in green and the CCD in red. Both spectra are averaged across the entire interface in Figure $1 \mathrm{~b}$. for both the K2 and the CCD spectra, the spectrometer was setup with a dispersion of $0.1 \mathrm{eV} /$ channel resulting in a spectrum of $400 \mathrm{eV}$ for the $\mathrm{K} 2$ and $200 \mathrm{eV}$ for the $\mathrm{CCD}$ in energy range. The $\mathrm{Ti} \mathrm{L}$ at $456 \mathrm{eV}$ is present in the spectrum extracted using the $\mathrm{K} 2$ but missing in the case of the CCD; b) comparison of the Fe L-edges spectra in the case of the K2 and the $\mathrm{CCD}$. The energy resolution in the case of the $\mathrm{K} 2$ spectrum is much higher, more features and details in the fine structure can be observed. 